\title{
Three-Dimensional Finite Element Ablative Thermal Response and Thermostructural Design of Thermal Protection Systems
}

\author{
John A.Dec ${ }^{*}$ \\ NASA Langley Research Center, Hampton, Virginia, 23681 \\ Robert D. Braun ${ }^{\dagger}$ \\ Georgia Institute of Technology, Atlanta, Georgia, 30332-0150
}

\begin{abstract}
A finite element ablation and thermal response program is presented for simulation of three-dimensional transient thermostructural analysis. The three-dimensional governing differential equations and finite element formulation are summarized. A novel probabilistic design methodology for thermal protection systems is presented. The design methodology is an eight step process beginning with a parameter sensitivity study and is followed by a deterministic analysis whereby an optimum design can determined. The design process concludes with a Monte Carlo simulation where the probabilities of exceeding design specifications are estimated. The design methodology is demonstrated by applying the methodology to the carbon phenolic compression pads of the Crew Exploration Vehicle. The maximum allowed values of bondline temperature and tensile stress are used as the design specifications in this study.
\end{abstract}

\section{Nomenclature}

1-D = one-dimensional

3-D $\quad=$ three-dimensional

$A D P \quad=$ advanced development program

$B_{i} \quad=$ pre-exponential factor for the $\mathrm{i}^{\text {th }}$ resin component

$c_{p} \quad=$ solid material specific heat, $\mathrm{J} / \mathrm{kg}-\mathrm{K}$

$C A D=$ computer aided design

$C A P=\mathrm{CEV}$ aerosciences program

$C E V \quad=$ crew exploration vehicle

$C F D=$ computational fluid dynamics

$C M A=$ aerotherm charring material ablation and thermal response program

$C T E=$ coefficient of thermal expansion

$D A C-3=$ design and analysis cycle 3

$E_{a i} \quad=$ activation energy for the $\mathrm{i}^{\text {th }}$ resin component, $\mathrm{J} / \mathrm{kg}$-mole

$F E A R=$ finite element ablation and thermal response design and analysis program

FIAT = fully implicit ablation and thermal response program

$h_{g} \quad=$ enthalpy of pyrolysis gas, $\mathrm{J} / \mathrm{kg}$

$H \quad=$ total enthalpy, $\mathrm{J} / \mathrm{kg}$

$\bar{h} \quad=$ weighted average solid enthalpy, $\mathrm{J} / \mathrm{kg}$

$i=$ node index, resin component index $(\mathrm{A}, \mathrm{B}, \mathrm{C})$

ITAR = international traffic in arms regulations

$\boldsymbol{k}=$ orthotropic thermal conductivity matrix, $\mathrm{W} / \mathrm{m}-\mathrm{K}$

$\dot{\boldsymbol{m}}_{g}^{\prime \prime} \quad=$ pyrolysis gas mass flux vector, $\mathrm{kg} / \mathrm{m}^{2}-\mathrm{s}$

\footnotetext{
* Aerospace Engineer, Structural and Thermal Systems Branch, MS 431, and AIAA Member.

$\uparrow$ David and Andrew Lewis Associate Professor of Space Technology, Guggenheim School of Aerospace Engineering, AIAA Fellow
} 


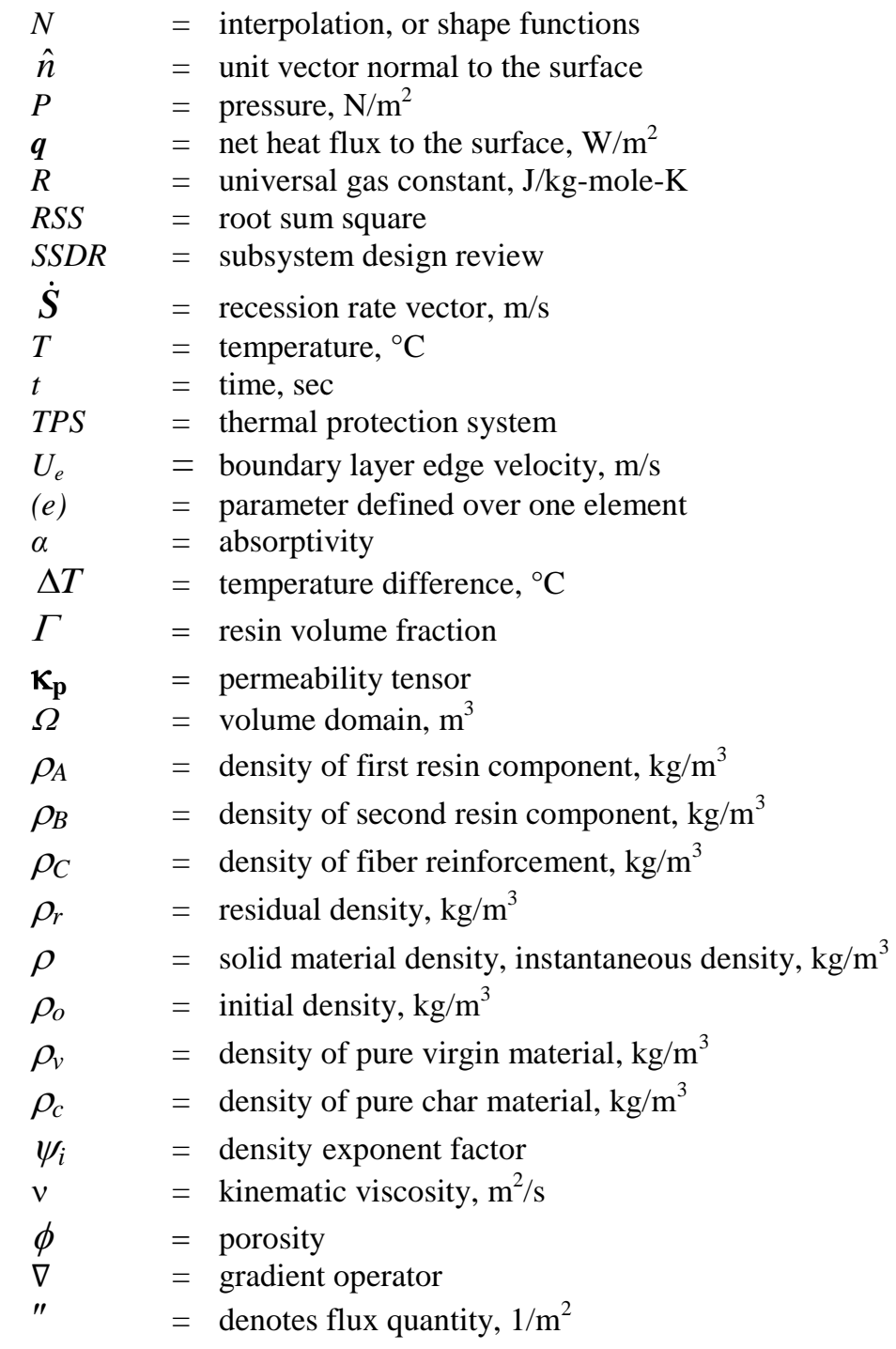

\section{Introduction}

ESIGN of a heatshield subsystem is multidisciplinary in nature; the heatshield design includes both the structural response to the flight loads, and the thermal response to the entry heating. Entry heating can also induce thermal stresses in the materials due to thermal expansion. To perform heatshield thickness sizing, the analyst relies on trajectory inputs from the guidance, navigation, and control team, aerodynamic heating distribution inputs from the aerothermodynamics team, and the geometric configuration from the mechanical design team. The analyst relies on the loads inputs from the loads and dynamics team, and the geometric configuration from the mechanical design team to perform the structural sizing. This design philosophy generally applies to heatshields and any other features found on the heatshield subsystem such as the compression pads found on the Orion Crew Exploration Vehicle (CEV).

The structural analyst generally takes advantage of the fact that the three-dimensional computer-aided design (CAD) geometry from the mechanical design team can be imported directly into their finite element analysis codes. Currently, there is no commercially available three-dimensional (3-D) thermal analysis code capable of performing high fidelity ablative thermal response analysis and sizing of heatshield materials. The thermal analyst typically uses the 3-D geometric configuration to create a one-dimensional (1-D) slice through the geometry at a specific point on the heatshield and performs a 1-D analysis using the fully implicit ablation and thermal-response (FIAT) ${ }^{1}$ program or some other analogous solution method. This 1-D analysis is repeated until the thickness is such that the sizing constraint, typically bondline temperature, is satisfied. The thermal analyst repeats this process for multiple locations on the heatshield to develop a thickness distribution, often without regard to any geometric constraints that 
exist. On a blunt body, sphere-cone type of vehicle, the points selected generally lie along radial lines emanating from the stagnation point to the vehicle shoulder. The mechanical design team is left to interpret these results and incorporate them into the system design. The analyst is generally unable to provide guidance in terms of how the design variables can be modified to meet geometric constraints and not exceed the thermal design specifications.

To perform a thermostructural analysis to assess thermal stresses, the structural analyst must interpolate the 1-D temperature data onto the larger 3-D structural model. Interpolating the thermal results from a limited set of points on the heatshield can cause sharp gradients when mapped to the structural model. These sharp gradients can lead to erroneous thermal stresses and cause the design team to modify the design in a suboptimal way.

Uncertainties exist in the parameters that are used as inputs to the thermal and structural response and yield uncertainty in the results of any subsequent analysis. Uncertainty in the numerical modeling of the associated physical phenomenon also contributes to the uncertainty of the results. Designing systems to meet operational requirements and design specifications under uncertain conditions is difficult. Factors of safety or margins are introduced to both the inputs and the results of the analysis to mitigate the effect of uncertainty. Applying a margin to the design has proven to be successful in the past, but often produces design solutions that are massive, suboptimal, and lacking in insight into the probability that a system will exceed the design specifications or meet the operational requirements.

The finite element ablation and thermal response (FEAtR, hence forth called FEAR) design and analysis program $^{2,3}$, has been developed and will be used to analyze the thermal response of an Orion CEV heatshield compression pad. For the structural response, the finite element equations for linear elasticity have been integrated into FEAR and the thermal stress is calculated simultaneously with the temperature. These analysis tools are then integrated into a novel design methodology where parameter sensitivities are performed, probabilistic ranges of the design variables are examined, and a Monte-Carlo simulation is performed where the probability of exceeding design specifications is assessed. In this paper, the FEAR program will be used to demonstrate a new thermal protection system (TPS) design methodology using the CEV heatshield compression pads as a design example.

\section{Mathematical Model}

The governing differential equations that describe the ablative thermal response problem consist of the conservation of mass, momentum, and energy. A detailed derivation of the governing differential equations from first principles is presented in Ref. 3 and summarized here. The conservation of mass may be written as

$$
\frac{\partial \rho}{\partial t}=-\nabla \dot{\boldsymbol{m}}_{g}^{\prime \prime}
$$

The instantaneous density is assumed to be a three-component mixture consisting of two resins and one reinforcing material components and is given by

$$
\rho=\Gamma\left(\rho_{A}+\rho_{B}\right)+(1-\Gamma) \rho_{C}
$$

The decomposition over time is calculated using an Arrhenius relation ${ }^{4,5}$ written as

$$
\frac{\partial \rho_{i}}{\partial t}=-\mathrm{B}_{i} e^{-E_{a_{i}} / R T} \rho_{o_{i}}\left(\frac{\rho_{i}-\rho_{r_{i}}}{\rho_{o_{i}}}\right)^{\psi_{i}} \quad i=A, B, C
$$

In general, ablative materials are porous materials and the porosity, as well as the permeability, increases as the material decomposes from the initial virgin state to a charred state. Therefore, Darcy's law is used to calculate the pressure drop due to the flow of pyrolysis gas through the material.

$$
\dot{\boldsymbol{m}}_{g}^{\prime \prime}=\frac{-\boldsymbol{\kappa}_{p}}{\nu \phi} \nabla P
$$

The conservation of energy for the conductive transport of energy through an ablative material that is decomposing in-depth may be written as

$$
\rho c_{p} \frac{\partial T}{\partial t}=\boldsymbol{k} \nabla T+\left(h_{g}-\bar{h}\right) \frac{\partial \rho}{\partial t}+\dot{\boldsymbol{m}}_{g}^{\prime \prime} \nabla h_{g}+\dot{\boldsymbol{S}} \rho c_{p} \nabla T
$$

American Institute of Aeronautics and Astronautics 


\section{Weak Formulation}

The corresponding weak form of the governing system of equations, (1), (4), and (5) may be constructed by first multiplying the residual of the differential equation by an appropriate set of weighting functions $\boldsymbol{W}$ and integrating by parts over the domain $\Omega$. If the weighting functions $\boldsymbol{W}$ are set equal to the shape functions $\boldsymbol{N}$ which approximate the unknown variables, the result is known as the Bubnov-Galerkin form ${ }^{6}$. The method of weighted residuals requires that the weighted average of the error, or residual, vanish over the solution domain. The weighted residual statement for the conservation of mass and momentum is given in Eqs. (6) and (7), respectively. The weighted residual statement for the conservation of energy is given in Eq. (8).

$$
\begin{gathered}
\iiint_{\Omega^{(e)}}\left[\nabla \dot{\boldsymbol{m}}_{g}^{\prime \prime(e)}-\frac{\partial \rho}{\partial t}\right] N_{i}^{P} d \Omega=0 \\
\iiint_{\Omega^{(e)}}\left[\dot{\boldsymbol{m}}_{g}^{\prime \prime(e)}+\frac{\boldsymbol{\kappa}_{p}}{v \phi} \nabla P^{(e)}\right] N_{i}^{u, v, w} d \Omega=0 \\
\iiint_{\Omega^{(e)}}\left[\boldsymbol{k} \nabla T^{(e)}+\left(h_{g}-\bar{h}\right) \frac{\partial \rho^{(e)}}{\partial t}+\dot{\boldsymbol{m}}_{g}^{\prime \prime} \nabla h_{g}^{(e)}+\dot{\boldsymbol{S}} \rho c_{p} \nabla T^{(e)}-\rho c_{p} \frac{\partial T^{(e)}}{\partial t}\right] N_{i} d \Omega=0
\end{gathered}
$$

In Eqs. (6) and (7), the shape functions that appear in the mass and momentum equations are different. In this formulation, the polynomial shape functions which approximate the pressure must be one order lower than those used to approximate the pyrolysis gas flux components in order to prevent an over constrained system of discrete equations. This makes the pressure discontinuous across element boundaries and thus pressure is not a primary variable in the weak formulation ${ }^{7}$.

Integrating Eqs. (7) and (8) by parts to eliminate the pressure derivative and reduce the derivative on the temperature to first order results in Galerkin's weak form for the conservation of mass, momentum and energy given by Eqs. (9), (10), and (11), respectively.

$$
\begin{gathered}
\iiint_{\Omega^{(e)}}\left[\nabla \dot{\boldsymbol{m}}_{g}^{\prime \prime}\right] N_{i}^{P} d x d y d z=\iiint_{\Omega^{(e)}} \frac{\partial \rho}{\partial t} N_{i}^{P} d x d y d z \\
\iiint_{\Omega^{(e)}}\left(\dot{\boldsymbol{m}}_{g}^{\prime(e)} N_{i}^{u, v, w}\right) d x d y d z+\iiint_{\Omega^{(e)}} \frac{\boldsymbol{\kappa}_{p}}{v \phi} \frac{\partial N_{i}^{u, v, w}}{\partial x} P^{(e)} d x d y d z=\iint_{\Gamma^{(e)}} N_{i}^{u, v, w}(P \cdot \hat{\boldsymbol{n}}) d \Gamma+\iint_{\Gamma^{(e)}} N_{i}^{u, v, w} V_{n} d \Gamma \\
\left.\iiint_{\Omega^{(e)}} k_{x} \frac{\partial T^{(e)}}{\partial x} \frac{\partial N_{i}}{\partial x}+k_{y} \frac{\partial T^{(e)}}{\partial y} \frac{\partial N_{i}}{\partial y}+k_{z} \frac{\partial T^{(e)}}{\partial z} \frac{\partial N_{i}}{\partial z}\right) d x d y d z+\iiint_{\Omega^{(e)}} \dot{\boldsymbol{m}}_{g}^{\prime \prime} \nabla h_{g}^{(e)} N_{i} d x d y d z \\
+\iiint_{\Omega^{(e)}} \dot{\boldsymbol{S}} \rho c_{p} \nabla T^{(e)} N_{i} d x d y d z-\iiint_{\Omega^{(e)}} \rho c_{p} \frac{\partial T^{(e)}}{\partial t} N_{i} d x d y d z+\iint_{\Gamma^{(e)}}(\mathbf{q} \cdot \hat{n}) N_{i} d \Gamma=0
\end{gathered}
$$

The surface integrals introduced by the integration by parts provide the connection to the surface boundary conditions present on any surface of the element. In the momentum equation, the first surface integral represents surface tractions and the second a specified mass flux at the surface. In the energy equation, the surface integral represents the net conductive flux into the volume, $\Omega^{(\mathrm{e})}$, from any surface bounded by $\Gamma$ and can be expanded to represent any number of boundary conditions by summation over all surfaces bounded by $\Gamma$. Since Eq. (11) is for one element, $\Gamma$ includes all of the surfaces of the element. For example, in the case of an ablative material, the surface boundary conditions are given by

$$
\iint_{\Gamma^{(e)}}(\mathbf{q} \cdot \hat{n}) N_{i} d \Gamma=\rho_{e} U_{e} C_{H}\left(H_{s r}-h_{s w}+B_{c}^{\prime} h_{c}+B_{g}^{\prime} h_{g}-B^{\prime} h_{w}\right)-q^{*}+q_{\substack{r a d \\ \text { out }}}-\alpha q_{\substack{\text { rad } \\ \text { in }}}
$$


The governing differential equations for linear elasticity are used to solve for the thermal stress and also to move the mesh while recession is occurring. These governing differential equations and corresponding weak form have been developed and described in detail in several references and are not presented in this paper ${ }^{6,8}$.

\section{Design Methodology}

The objective of this design methodology is to link the heatshield design (or any other heatshield feature) to the thermostructural analysis. The link to the analysis includes both the thermal and structural response. For a heatshield, or compression pad application, the analysis parameters of interest, which could also be thought of as the driving design specifications, are the bondline temperature and the maximum thermal stress. The link from the analysis to the design comes from understanding the variation in the thermostructural response over the range of the design variables.

A link from the design to the fluid flow analysis is not directly made here. The link from the design to the flow environment represents an important aspect of heatshield and compression pad design but is beyond the scope of this study and is a topic for future work. For this work, it is assumed that a high fidelity computational fluid dynamic (CFD) solution for the flow field exists at several discrete time points along the entry trajectory for the threedimensional entry vehicle. It is further assumed that the heating from the entry trajectory code has been modified to match the results of the CFD at those discrete time points. Additionally, it is assumed that any augmented heating near any heatshield feature has been calculated and exists in the form of spatially distributed scale factors, also known as bump factors. These bump factors are simply multipliers to the smooth body heating.

Uncertainties in the CFD calculations and in the trajectory are also assumed to exist. In this study, the heating and trajectory will be inputs to the analysis and already include their associated uncertainties. Using the bondline temperature and thermal stress as the measure, sensitivities to the analysis input parameters provide insight into how much the uncertainty in each input contributes to the response of the system. Moreover, characterizing the sensitivity to each input helps determine which uncertainties and input parameters should be included in a Monte Carlo simulation. Performing a sensitivity study is the first step in the design process. Since each system and corresponding analyses are different, the sensitivity analysis cannot be omitted and the input parameters chosen for the analysis will be system and problem dependant.

The next step is to examine how the thermostructural response varies over the ranges of the design variables. The objective is to discretize the design space by selecting discrete points for each design variable from within the range of the design space variation. For example, if the ply angles in the layup of the ablative heatshield were a variable of interest, breaking the layup angle into three levels one could choose the minimum, maximum, and a value in the middle of the range. Once the design space has been discretized, a matrix of designs is created where each one represents a different combination of the design variables. The process of systematically varying the design variables is known as design of experiments and there are many ways the process can be performed. In this study, a full factorial scheme will be utilized since this scheme covers all possible combinations in the discretized space, albeit at the expense of having to run a maximum number of cases in the analysis. Other schemes such as the central-composite design reduce the number of runs, but at the expense of not using all possible combinations of the design variables. FEAR is run for each design in the matrix and the bondline temperature and thermal stress are recorded.

After the matrix of designs has been analyzed, a contour plot showing the variation in bondline temperature over the design space is generated. Within the contour of bondline temperature, any design constraints present can be superimposed on the contour. Utilizing the plot, the optimum design based on minimizing the objective function, which in this case is the bondline temperature, can be determined. In this case, with the bondline temperature as the objective function, the thermal stress design specification is plotted as a constraint. Conversely, if the thermal stress were chosen as the objective function, the bondline temperature would be plotted as a constraint.

To design for uncertainty, the input parameters and their associated uncertainties must be incorporated into the design and analysis. The most influential uncertainties, as determined by the sensitivity study, are selected for use in a Monte Carlo simulation. Based on minimizing the objective function, subject to the constraints of the problem, the optimum design established above, is the candidate design that the Monte Carlo simulation is performed on. From the results of the simulation, the probability that the design will exceed the design specification can be quantitatively determined. A histogram showing the distribution of the bondline temperature and thermal stress can be generated and the 99\%, 95\%, and 90\% bondline temperature and thermal stress can be displayed. If the probabilities are satisfactory then the process is complete. If they are not satisfactory, the constraints can be modified and the process 
of finding the optimum design and running the Monte Carlo are repeated. The design process is summarized in Table 1.

Table 1. Design process procedure

\begin{tabular}{ll}
\hline \hline \multicolumn{1}{c}{ Design Process } \\
\hline 1 & Perform sensitivity study \\
2 & Discretize design space \\
3 & Perform design of experiments \\
4 & Analyze matrix of designs \\
5 & Determine optimum design \\
6 & Perform Monte Carlo simulation on optimum design \\
7 & Determine probabilities \\
8 & Check for satisfactory probabilities; if unsatisfactory \\
\hline \hline
\end{tabular}

\section{Orion CEV Compression Pad Design}

The Orion Crew Exploration Vehicle is first entry vehicle designed by NASA to have a man-rated ablative heatshield since the Apollo program. Like Apollo, the vehicle includes six hard-point compression pads that must transfer the launch loads to the command module during launch as well as survive the thermal environment during re-entry.

There is a striking similarity between the Apollo and the Orion command modules. However, the Apollo Program had a different set of design requirements and a different risk posture than the Orion Program. The Orion Program has tasked the engineers with quantifying the risks associated with the design of each system. One of the most scrutinized systems is the heatshield. There is no redundancy built into the heatshield system; the system must work and must work even under off-nominal operating conditions. The use of legacy heatshield sizing tools such as Aerotherm's charring material ablation (CMA) and thermal response program and FIAT provide no insight into the ability of the heatshield to perform its function under off-nominal environmental conditions or variable material properties.

The design approach described in this section will be utilized to answer the question of how the compression pad performs under varying conditions by incorporating the uncertainty in the input parameters. In addition, the push to reduce the heatshield mass fraction must be considered. Simply adding heatshield thickness, which directly translates into mass, to increase the robustness of the design is not generally an acceptable system-level solution. Given this desire, the current 1-D ablative thermal analysis and design process is inadequate, especially when considering the Orion heatshield compression pads. The compression pad subsystem is a complex 3-D system which interfaces with many different materials. The boundary conditions near the compression pad are complex, and spatially varying in the plane normal to the external surface of the compression pad, as shown in Fig. 1.

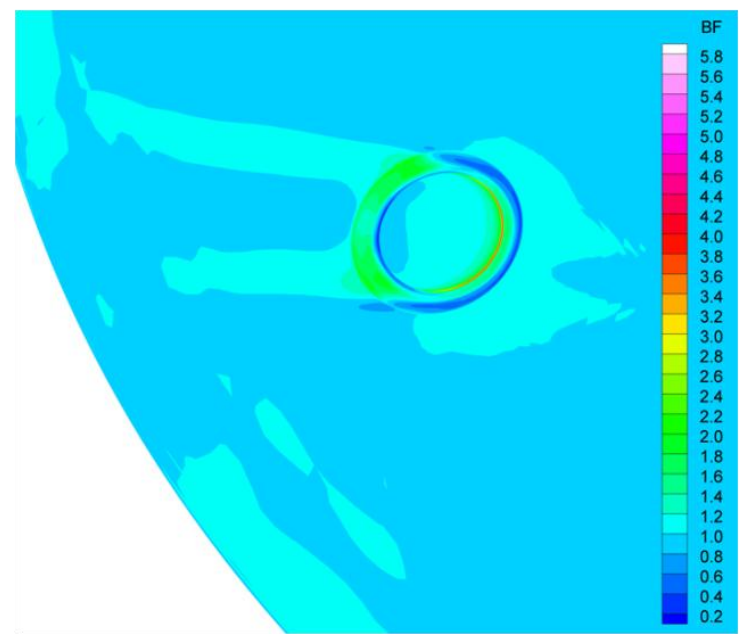

Fig. 1 Heating augmentation bump factors near the $120^{\circ}$ compression pad location. ${ }^{9}$ 
The Orion CEV smooth body heating reaches a peak combined convective and radiative heat rate of $829 \mathrm{~W} / \mathrm{cm}^{2}$. The heating augmentation factors shown in Fig. 1 are applied to the smooth body convective heating rate only. The trajectory used in this study comes from the third Orion CEV Design and Analysis Cycle (DAC-3) lunar return profile and is plotted in Fig. 2. The nominal trajectory heating is anchored to a CFD solution at discrete time points and is for a smooth body vehicle which does not account for any shape change. The complexity of the heatshield compression pads, the requirements placed on them, and the complex boundary conditions around them provides the motivation for this study.

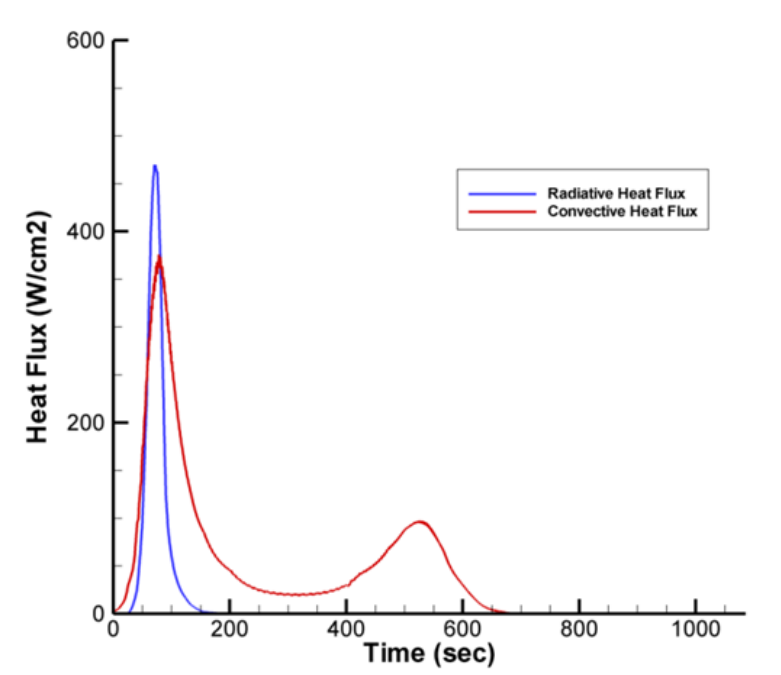

Fig. 2 CEV DAC-3 lunar return trajectory heating near the $120^{\circ}$ compression pad.

The Orion penetration subsystem is made up of six reinforced hard points on the forebody TPS. Each hard point consists of an MX4926N carbon phenolic compression pad and an inconel tension tie. The MX4926N plies are inclined $20^{\circ}$ with respect to the bottom of the pad and aligned with the centerline flow streamline. The compression pad is inserted into the titanium carrier structure, then the acreage Avcoat TPS is built up around the compression pad. There is a $20^{\circ}$ bevel cut into the Avcoat both to provide clearance for the service module connection and to minimize the effects of heating augmentation. A tension tie rod passes through the center of the pad and connects directly to the vehicle structure. The tension tie rod is preloaded to balance the weight of the vehicle plus added factors of safety. The preload is distributed over the six compression pad/tension tie rod assemblies. The compression pad is bonded and bolted to a titanium carrier structure. There is a small gap between the compression pad and the Avcoat and the gap is filled with a compliant gap filler material. The gap reduces the stress in the surrounding materials by allowing for a small amount of differential thermal expansion. A cross section of the Orion heatshield compression pad with the surrounding titanium structure and Avcoat heatshield is shown in Fig. 3 where the tension tie rod has been removed for clarity. For analysis purposes, the bondline is the bottom surface of the compression pad and titanium insert interface.

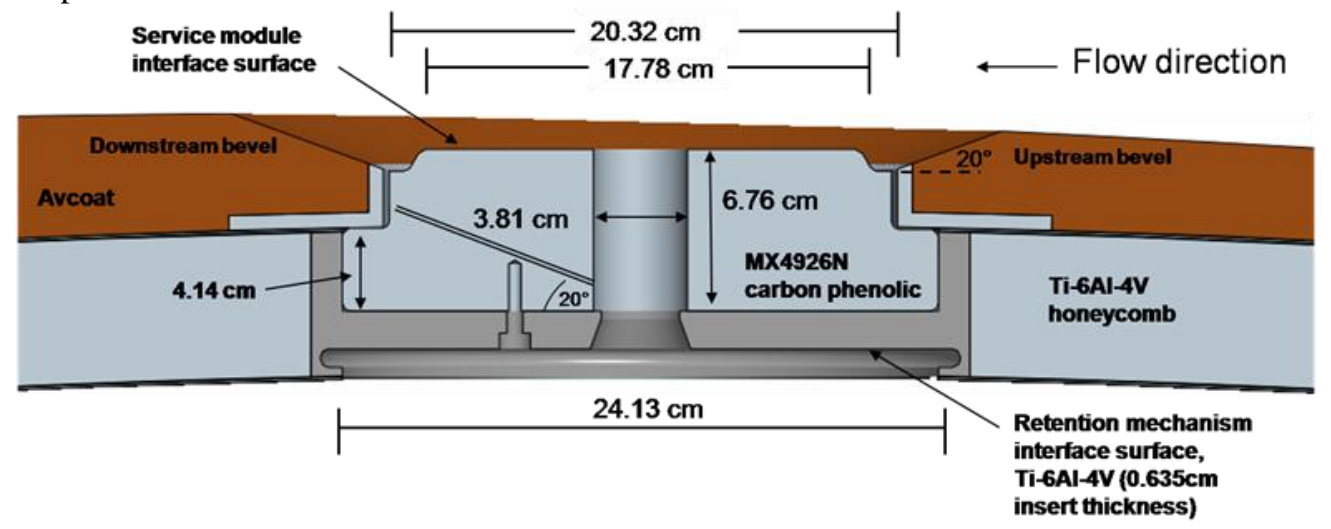

Fig. 3 Cross section of the DAC-3 Orion heatshield compression pad, surrounding structure, and acreage heatshield. 
The compression pad design, with the surrounding structure, and TPS have a complex geometric shape. The geometry along with a distributed heating boundary condition shown in Fig. 1, and anisotropic material properties makes this system difficult to characterize with a 1-D analysis.

\section{A. Thermal Response Sensitivity to Material Properties}

The first step in the design process is to perform a sensitivity analysis to characterize how variations in the material properties affect the thermostructural response. Along with the properties examined, each of the associated $3-\sigma$ uncertainties are listed in Table 2.

Table 2. Sensitivity study properties and associated uncertainties.

\begin{tabular}{lc}
\hline \hline \multicolumn{1}{c}{ Property $^{\dagger}$} & $3 \sigma$ Uncertainty \\
\hline Virgin thermal conductivity & $\pm 0.45 \mathrm{~W} / \mathrm{m}-\mathrm{K}$ \\
Char thermal conductivity & $\pm 0.88 \mathrm{~W} / \mathrm{m}-\mathrm{K}$ \\
Virgin specific heat & $\pm 19.1 \mathrm{~J} / \mathrm{kg}-\mathrm{K}$ \\
Char specific heat & $\pm 30.7 \mathrm{~J} / \mathrm{kg}-\mathrm{K}$ \\
Virgin density (room temperature) & $\pm 6.3 \% \mathrm{~kg} / \mathrm{m}^{3}$ \\
Char emissivity & \pm 0.06375 \\
Virgin heat of formation & $\pm 15 \% \mathrm{~J} / \mathrm{kg}$ \\
Pyrolysis gas enthalpy & $\pm 15 \% \mathrm{~J} / \mathrm{kg}$ \\
Permeability & $\pm 10.2 \% \mathrm{~cm}{ }^{2}$ \\
Porosity & $\pm 8.7 \%$ \\
Coefficient of thermal expansion & $\pm 46.17 \% \mathrm{~m} / \mathrm{m} /{ }^{\circ} \mathrm{C}$ \\
Compressive modulus & $\pm 28.98 \% \mathrm{~Pa}$ \\
\hline \hline
\end{tabular}

${ }^{\bar{p} \text { Uncertainties calculated in the database are given as functions of temperature and principle }}$ direction. They are given as average values here unless otherwise noted.

The uncertainties listed in Table 2 are for the MX4926N carbon phenolic. Material property data for the MX4926N carbon phenolic was obtained from an extensive database developed by ATK Launch Systems and NASA. ${ }^{10}$ The data in this report falls under the purview of the U.S. Munitions List as defined in the International Traffic in Arms Regulations (ITAR) and therefore cannot be presented here. For this material property sensitivity study heating augmentation was not applied to the compression pad model.

Using these property variations FEAR was run 24 times where in each run a single property was set to its minimum or maximum 3- $\sigma$ uncertainty which isolated that property as being the only factor influencing the bondline temperature and thermal stress. It is assumed that the variations in the properties are independent and that the corresponding change in material response is due solely to that property being changed. Property independence can be determined and a more sophisticated sensitivity can be performed, but is beyond the scope of the current work. Results of the material property sensitivity are shown in Fig. 4 and Fig. 5. In both Fig. 4 and Fig. 5, the $-3 \sigma$ bars are the first in the pair for each property. The bondline temperature is most sensitive to the variations in the virgin and char thermal conductivity, and to a lesser extent by the virgin density and virgin heat of formation variations. The pyrolysis gas enthalpy, char emissivity, and the virgin specific heat have only a minor influence on the bondline temperature influencing it less than $1.0 \%$. The compressive modulus, permeability, porosity, and coefficient of thermal expansion (CTE) have little to no affect on the bondline temperature each influencing the bondline temperature less than $0.20 \%$. The across-ply tensile stress is most sensitive to the CTE, compressive modulus, and virgin thermal conductivity. All other property variations produced less than a $2.5 \%$ change in the across-ply tensile stress. As a result of the material property sensitivity study, the input parameters having the most influence on the design specifications have been identified for use in step 6 of the process outlined in Table 1 . 


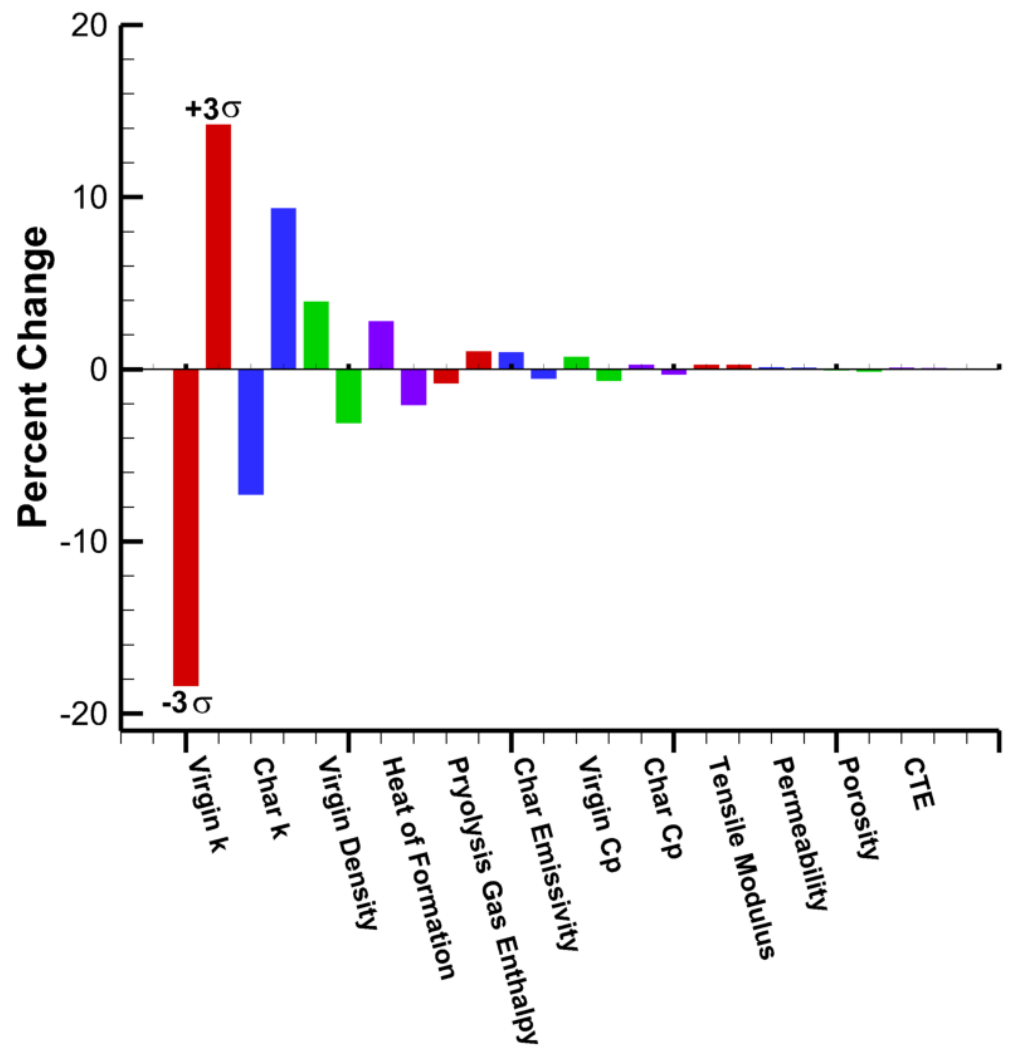

Fig. 4 Bondline temperature sensitivity.

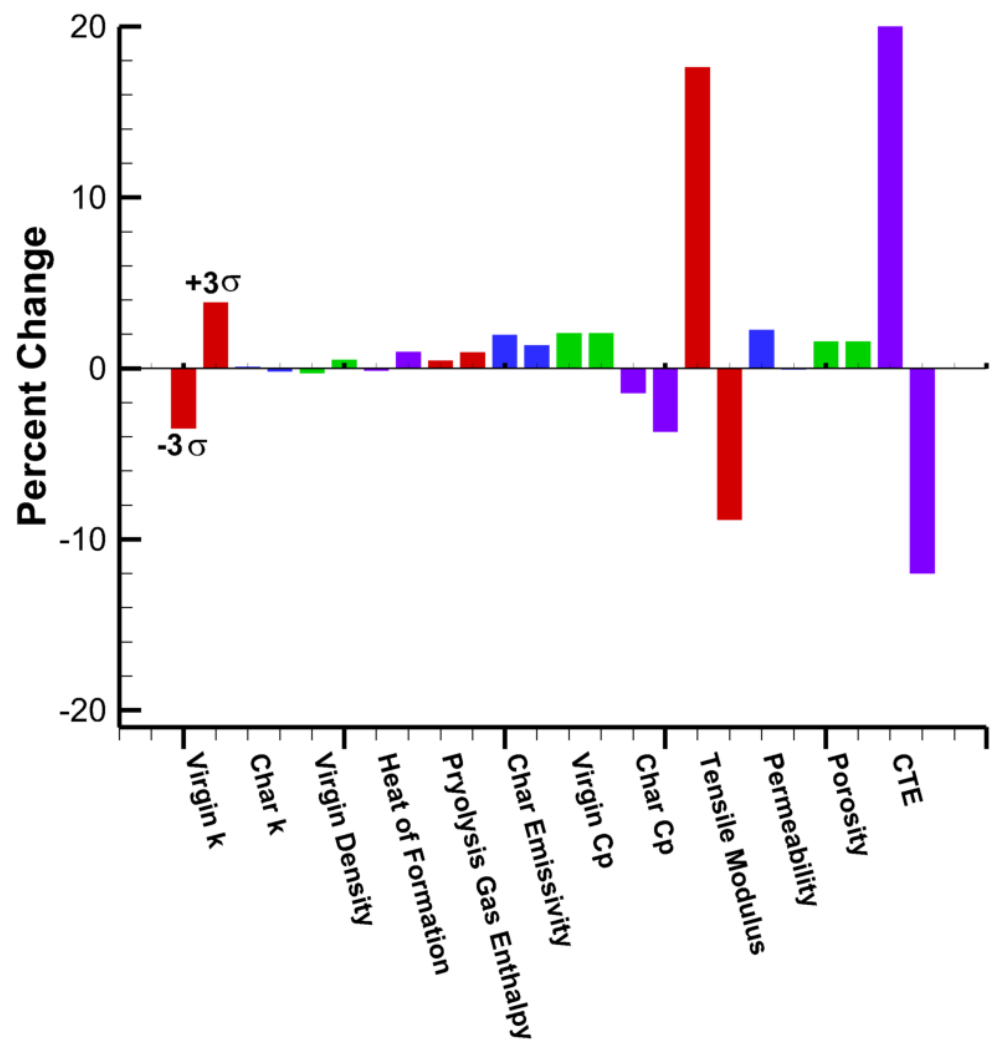

Fig. 5 Across-ply tensile stress sensitivity. 


\section{B. Deterministic Analysis}

The second through fifth steps in the design process, summarized in Table 1, involve deterministic analyses of the compression pad. Prior to this analysis a baseline analysis of the "as-designed" compression pad must be performed for comparison purposes.

\section{1. "As-Designed" Compression Pad Analysis}

The 1-D compression pad sizing for the DAC-3 lunar return entry environment was performed by the CEV TPS Advanced Development Project (ADP) compression pad team. ${ }^{11}$ The sizing was performed without considering the affects of heating augmentation. The compression pad design at the TPS Subsystem Design Review (SSDR) is shown in Fig. 3 and was the design used for this study. Since that time, the design of the compression pad subsystem has evolved, however, for the purposes of demonstrating the current design methodology, the SSDR design is sufficient. The mass of the as-designed compression pad and titanium insert system was $5.39 \mathrm{~kg}$. The boundary conditions applied to the compression pad are shown in Fig. 6. The heating augmentation factors are mapped to the FEAR mesh from the CEV Aerosciences Project (CAP) team's aeroheating database using Tecplot's internal inverse distance interpolation routine. ${ }^{12}$
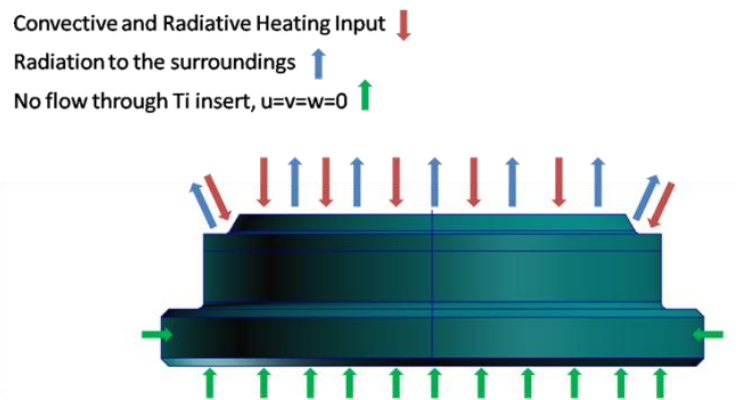

Fig. 6 Compression pad boundary conditions.

The augmentation factors used in the analysis are shown in Fig. 1 and the result of the mapping can be visualized in either Tecplot ${ }^{\circledR}$ of MSC PATRAN ${ }^{\circledR}$. The mapped augmentation factors are shown from within PATRAN in Fig. 7. The coarse circumferential mesh of the compression pad provides a low resolution of the augmentation factors on the FEAR mesh. The augmentation factors were mapped without regard to whether total heat load was preserved, future work will include a mapping method which does preserve total heat load. For this study the low resolution in the augmentation factor mapping is a current limitation that must be endured.
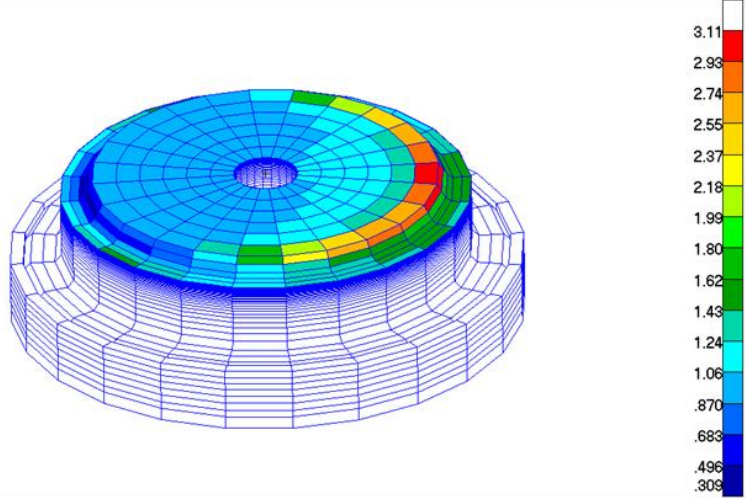

Fig. 7 Heating augmentation factors mapped to FEAR.

The resultant temperature distribution through the compression pad at peak heating and at the end of the trajectory is given in Fig. 8 and Fig. 9, respectively. The bondline temperature limit for the compression pad is $315.2^{\circ} \mathrm{C}$, and the maximum bondline temperature even under augmented heating conditions is $152.8^{\circ} \mathrm{C}$. 


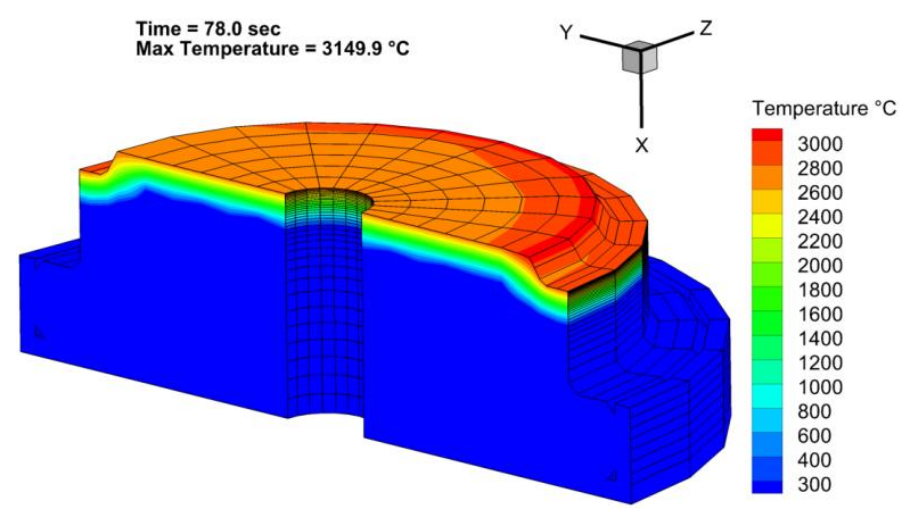

Fig. 8 Compression pad temperature distribution 4 seconds after peak heating.

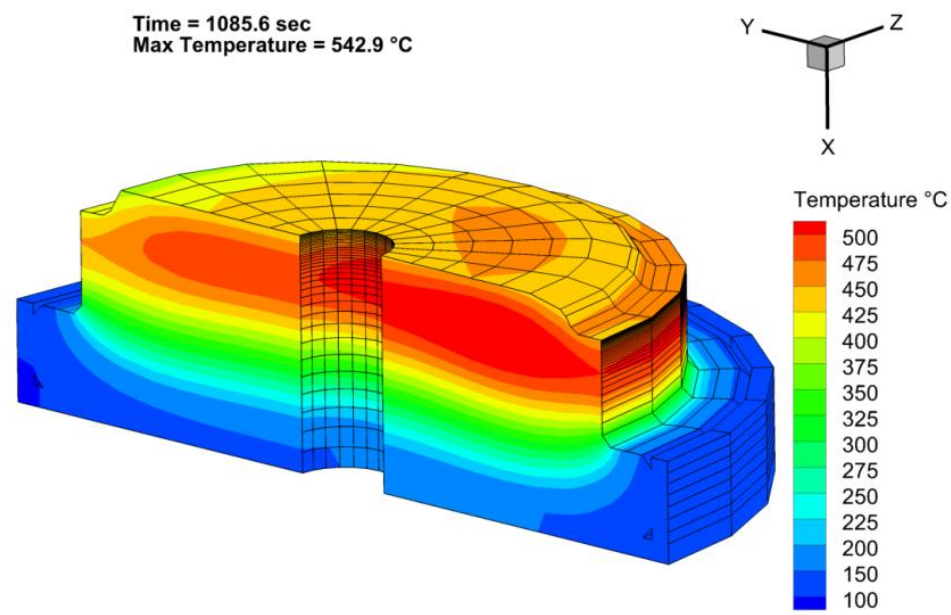

Fig. 9 Compression pad temperature distribution at parachute deployment.

There are several reasons which demonstrate the need for a three-dimensional analysis of the pad and a new design methodology. The first reason has to do with the current design philosophy and way the 1-D sizing for the compression pad is performed. The pad is sized using FIAT with three different cases. The first sizing case uses the nominal smooth body heating and applies trajectory dispersion factors to the convective and radiative heating. The second case uses the smooth body heating and applies the trajectory dispersions factors and factors due to the uncertainty in the aeroheating CFD calculations. The third case uses the same environment as the first case, but this time the pad is sized to a bondline temperature of $255.5^{\circ} \mathrm{C}$, reducing the bondline limit is meant to simulate the uncertainty in calculating the thermal response of the material. The third case is the most limiting in terms of sizing, however, the second case represents the highest heating and total heat load. The thickness that results from these sizing cases are root sum squared together and then an additional amount of thickness is added to account for uncertainty in the amount of recession being calculated. This becomes the minimum design thickness necessary to prevent the bondline temperature from being exceeded. Additionally, $0.889 \mathrm{~cm}$ is added to create a feature for the Service Module connection to transfer the shear loads into the compression and the rest of the Command Module. For the analysis performed in this study, the case 2 trajectory and aerodynamic heating is used since they produce the highest surface temperatures and most thermal soak back into the pad. As the FEAR analysis has shown the bondline temperature does not approach violating the temperature limit which suggests the current Orion design methodology is conservative.

In addition to the current deign practice; there are two main effects that the 1-D analysis cannot capture. The first effect is the fact that the in-plane virgin thermal conductivity of the compression pad material is roughly $35 \%$ greater than the through-the-thickness thermal conductivity. The in-plane char thermal conductivity is $36-50 \%$ greater depending on the temperature. Even though there is heating augmentation present, the higher in-plane conductivity tends to spread the heat out across the compression pad and away from the bondline. The second main 
effect not captured by the 1-D sizing analysis is the 3-D geometric effect of having extra mass not directly in the path of the surfaces that are being heated. The area in question is highlighted with a circle in Fig. 10.

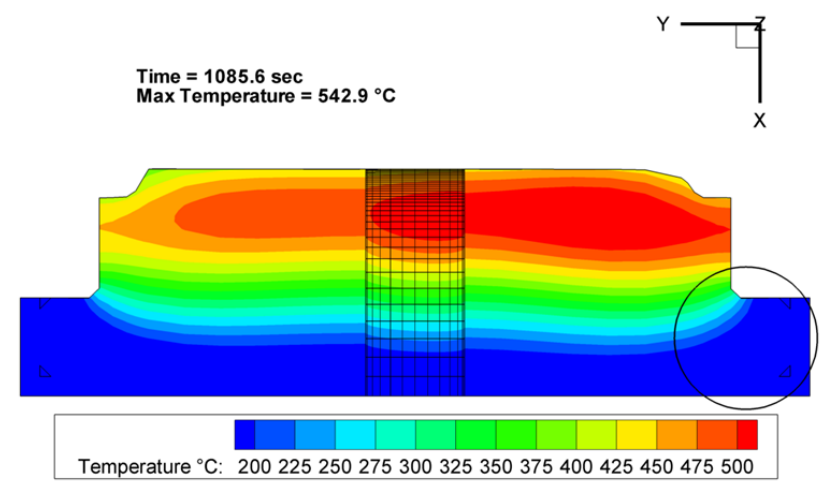

Fig. 10 Region of increased mass of the compression pad.

There is additional compression pad material and the side wall of the titanium insert in this region; both provide additional thermal mass and serve to regulate the heat being soaked towards the bondline. The CEV TPS ADP compression pad team designated this region as the "top hat" feature. The side which has the circle is also the side that has the highest heating augmentation factors. On the augmented heating side, the heat penetrates deeper into the top hat region, but the temperature towards the bondline is no more severe than the side without the augmented heating. The temperature equalization is due to the extra thermal mass absorbing energy and the in-plane thermal conductivity allowing conduction in this direction. Had heating augmentation been applied to a 1-D sizing analysis, the extra heat from the augmentation would be conducted directly to the bondline and would have given an incorrect higher bondline temperature.

FEAR also computes the thermal stress during the simulation. The interval for stress computations is set by the user and in this case was set to 5 second intervals. It has been shown that the most critical stress in the MX4926N material is the across-ply tensile stress. ${ }^{10,13}$ The criticality of across-ply tensile stress is mainly due to the fact that there are no reinforcing fibers in-between plies and that the majority of the strength in this direction comes from the phenolic resin which is generally lower than the reinforcing fiber. In this study, the across-ply tensile stress will be tracked as the stress design specification.

There are many different opinions on the subject of when a charring material is considered to have failed. There is a belief that if the allowable stress is exceeded at any time, whether high stress is in the char or virgin material, then there is a failure. There is also a belief to ignore high stresses that occur in the char material all together. Another complicating factor is that the allowable stress for the material is a function of temperature. For these reasons, failure of the MX4926N material will not be assessed in this study, the goal will be to minimize the acrossply stress without adversely affecting the stresses in the other directions and violating other imposed constraints.

The peak across-ply tensile stress occurs several seconds after peak heating at 150 seconds into the trajectory and is given in Fig. 11. The density distribution through the compression pad at 150 seconds is given in Fig. 12. The peak tensile stress is occurring where all the material has been converted to char and that the peak compressive stress is occurring at the leading edge of the pyrolysis zone.

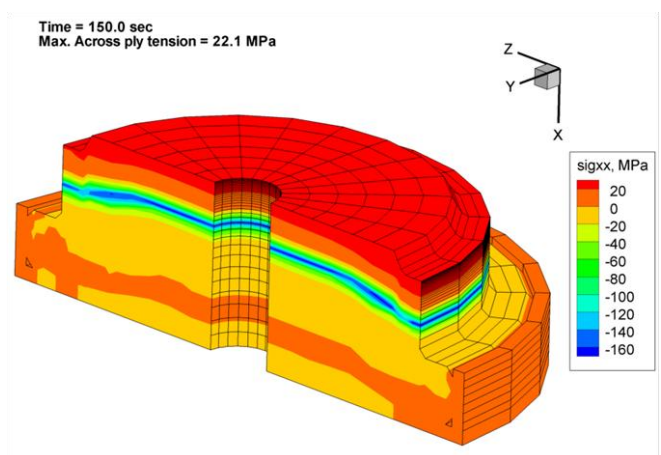

Fig. 11 Peak across-ply thermal stress at 150 seconds.

12

American Institute of Aeronautics and Astronautics 


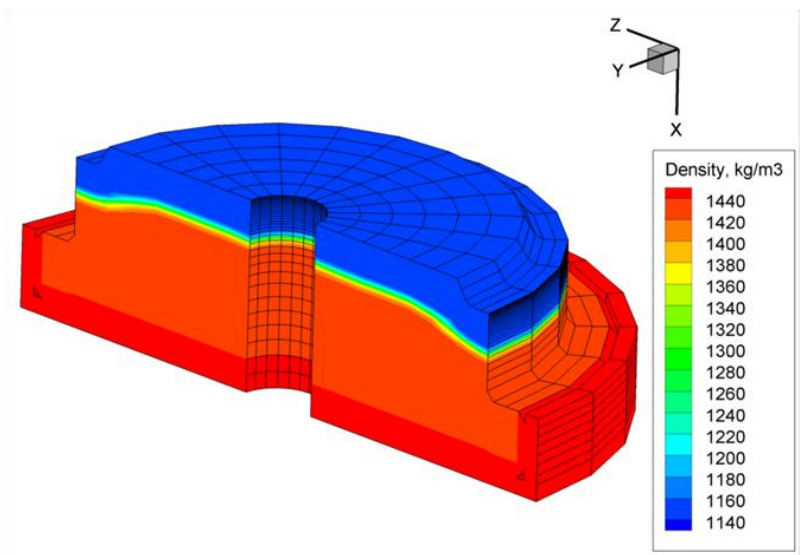

Fig. 12 Density distribution at 150 seconds.

\section{Optimum Compression Pad Design Analysis}

Once the baseline design has been analyzed, the objective is to optimize the design. Optimization is the fifth step in the design process outlined in Table 1. Prior to proceeding to this step, the pertinent design variables must be identified, valid ranges for them determined and then they are discretized. The design variables of interest for the Orion CEV compression pads are the pad thickness, material ply layup inclination angle, and the titanium insert structure thickness behind the pad. These are only a small subset of analysis parameters and design variables available to the designer; many others may be included in the methodology. However, for demonstration purposes, the selection will be limited to these three. Using engineering judgment and general knowledge of the design, the design variable values that would produce viable designs are defined and summarized in Table 3 .

Table 3. Design variable range.

\begin{tabular}{lcc}
\hline \hline \multicolumn{1}{c}{ Design Variable } & Minimum & Maximum \\
\hline Compression pad thickness $(\mathrm{cm})$ & 5.486 & 8.026 \\
Titanium insert thickness $(\mathrm{cm})$ & 0.254 & 1.270 \\
Ply angle (degrees) & 0.0 & 90.0 \\
\hline \hline
\end{tabular}

Once the design variable ranges have been defined, the process of discretizing the variable ranges can begin. Generally, it is not known a priori how many levels each of the design variables should be broken into. A rule of thumb would be to discretize the design variables into as few levels as possible in order to minimize the number of runs required, examine the results, and then add levels based on how each design variable affects the design specifications being tracked, which in this case would be the bondline temperature and across-ply tensile stress. The initial discretization for the design variables listed in Table 3 is three levels each. Upon examining the results, it is determined that the across-ply tensile stress is a strong function of ply angle, so the ply angle is broken down further so that the ply angle has seven levels. The design variable values are coded so that they vary between -1 and +1 . Since the scale of each variable is significantly different, coding the variables in this way normalizes the variables with respect to one another and aids in the visual display of the results. The discrete values used and the corresponding coding are listed in Table 4.

Table 4. Design variable discretization.

\begin{tabular}{lccccccc}
\hline \hline & \multicolumn{7}{c}{ Coded Values } \\
\cline { 2 - 8 } \multicolumn{1}{c}{ Design Variable } & -1.0 & -0.667 & -0.333 & 0.0 & 0.333 & 0.667 & 1.0 \\
\hline Pad thickness (cm) & 5.486 & ---- & ---- & 6.756 & ---- & ---- & 8.026 \\
Ti insert thickness (cm) & 0.254 & --- & --- & 0.762 & --- & --- & 1.270 \\
Ply angle (degree) & 0.0 & 15.0 & 30.0 & 45.0 & 60.0 & 75.0 & 90.0 \\
\hline \hline
\end{tabular}

The 3 by 3 by 7 full factorial design of experiments required 63 runs of FEAR to define the variation of the bondline temperature and across-ply tensile stress over the ranges of the design variables. A contour plot showing

13

American Institute of Aeronautics and Astronautics 
the variation of the bondline temperature with the design variables is shown in Fig. 13. The bondline temperature varies non-linearly with the design variables in Fig. 13. Also shown on Fig. 13 are the known constraints of the compression pad and titanium insert system.

The constraints can be categorized into three groups: material limitation, geometric configuration, and mission, or project management imposed. Generally, most of the constraints will fall into the material limitation category like the maximum bondline temperature, the maximum across-ply stress, and ply angle. Often there are mission, or project management imposed constraints. System mass usually falls into this category since mass is a premium on any spacecraft. Sometimes there are configuration specific geometric constraints like the maximum total allowable thickness for the compression pad and titanium insert. On the Orion CEV TPS, this is a real geometric constraint that must be met in order to allow a common service module interface height at every pad location and to avoid a forward facing step with respect to the upstream Avcoat TPS due to differential recession between the two.

Including the constraints on the bondline temperature contour shows how they affect the possible combinations of design variables and shows which combinations are viable. The constraints make finding the optimum somewhat easier since some combinations of the design variables can be eliminated immediately.

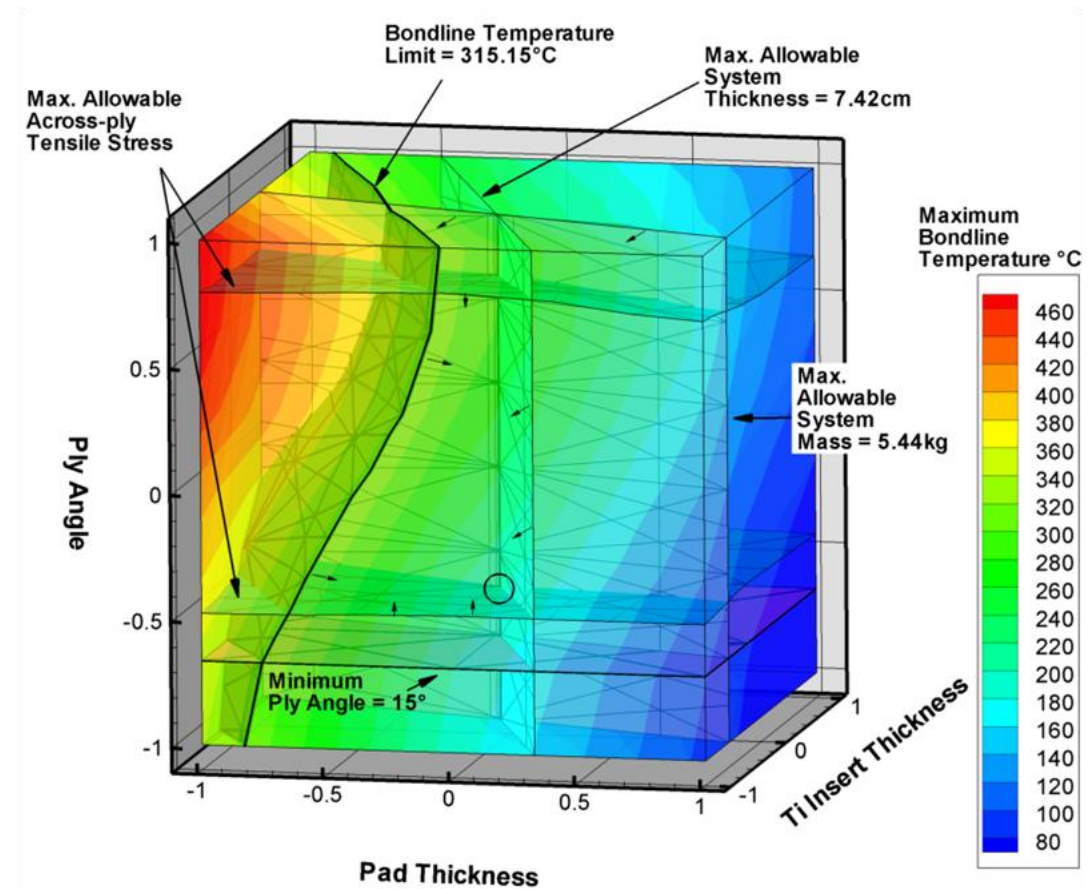

Fig. 13 Compression pad bondline temperature variation with imposed constraints.

The contour plot shows the viable design space is bounded by the maximum bondline temperature, maximum across-ply tensile stress, maximum system mass, and maximum total system thickness. Finding the optimum design can be accomplished either numerically or by selecting the values of the design values off of the contour plot. In this example, the contour plot was used to get a reasonable estimate for the design variables which represented the starting point in the numerical determination of the optimum compression pad design. The starting point is shown on Fig. 13 as a small circle at the intersection of the mass, stress, and total thickness constraints.

From the data generated from the 63 run full factorial design of experiments, a linear least squares fit of the bondline temperature was generated as a quadratic function of all the design variables. The across-ply stress was also fit with a linear least squares fit and was found to be best represented by a sixth order polynomial which was quadratic in pad thickness and sixth order in ply angle. The system mass was a simple linear function of the pad and titanium insert thicknesses. The ply angle and total thickness constraints were constants.

A metric, or target value for the across-ply tensile stress must be established, since no assessment of failure is being made in terms of the across-ply tensile stress. The metric chosen to improve the as-designed compression pad is that the across-ply tensile stress must be less than the $22.1 \mathrm{MPa}$ calculated in the as-designed compression pad and that no more than a $10 \%$ increase in the stresses in the other directions could be tolerated. 
Using a spreadsheet, a simple constrained optimization problem was set up and run. The result of the optimization was that the pad thickness should be $6.706 \mathrm{~cm}$, the titanium insert thickness $0.655 \mathrm{~cm}$, and the ply angle $26^{\circ}$. The ply angle was rounded up to the nearest degree since it is not practical to control the ply angle to the tenth of a degree, doing so introduces manufacturing inconsistencies and can lead to material failures. ${ }^{13}$ The mass of the compression pad and titanium insert system was increased slightly to $5.43 \mathrm{~kg}$; a $0.74 \%$ increase in mass compared to the as-designed compression pad. This increase is deceiving since the optimized pad analysis included heating augmentation. To make a better comparison, the 1-D root sum square (RSS) sizing process was performed again, this time with heating augmentation included. The result of performing the 1-D sizing with heating augmentation is that the required pad thickness would have to increase to $8.407 \mathrm{~cm}$ making the pad $25.36 \%$ thicker than the optimized pad. The mass of the 1-D sized pad with heating augmentation would be $6.14 \mathrm{~kg}$ making it $13.1 \%$ heavier than the optimized pad.

The bondline temperature for the optimized pad was calculated to be $169.9^{\circ} \mathrm{C}$ which is an increase of $17.1^{\circ} \mathrm{C}$ compared to the as-designed compression pad. Compared to the pad sized with heating augmentation, the bondline temperature increased $95.1^{\circ} \mathrm{C}$. This suggests that following the current design practice and performing a 1-D sizing for geometry that has 3-D features is highly conservative.

The maximum across-ply tensile stress was reduced by $19.9 \%$ to $17.7 \mathrm{MPa}$ compared to the as-designed compression pad. The design variables and the objective functions (or design specifications), for the baseline compression pad design, the baseline design including heating augmentation (determined by 1-D analysis), and the optimum design are summarized in Table 5. What remains to be determined is how these design changes affect the probabilities of exceeding the design specifications of bondline temperature and tensile stress which are the last three steps in the design process.

Table 5. Design variable and specification comparison.

\begin{tabular}{lccc}
\hline \multirow{2}{*}{ Design Variable } & \multicolumn{2}{c}{ Current 1-D Sized Compression Pad } \\
& Design & 3-D Optimized \\
& Baseline & $\begin{array}{c}\text { w/Heating } \\
\text { Augmentation }\end{array}$ & Design \\
& & 8.407 & 6.706 \\
\hline Compression Pad Thickness (cm) & 6.782 & 0.635 & 0.655 \\
Titanium Insert Thickness (cm) & 0.635 & 20 & 26 \\
Ply Angle (degrees) & 20 & 6.14 & 5.43 \\
Mass (kg) & 5.39 & 74.8 & 169.9 \\
Max. Bondline Temperature $\left({ }^{\circ} \mathrm{C}\right)$ & 152.8 & 22.1 & 17.7 \\
Max. Across-ply Tensile Stress (MPa) & 22.1 & & \\
\hline \hline
\end{tabular}

\section{Probabilistic Analysis}

The optimum compression pad design which minimizes the bondline temperature and satisfies all the constraints, determined in Section V, B2, is then used in the Monte Carlo simulation. The final steps in the design process outlined in Table 1 are to determine the probabilities that the bondline temperature and the across-ply tensile stress target are exceeded. A Monte Carlo simulation is performed on the optimum design to determine the probabilities. Going back to the material property sensitivity study, the top six properties influencing the thermostructural response are identified. These six properties are varied randomly according to an assumed Gaussian distribution. Other distributions are possible and may better represent a particular property, but for simplicity, all properties are assumed to follow Gaussian distributions.

A 500 sample Monte Carlo simulation was run on an Intel $2.93 \mathrm{GHz}$ Xeon quad core processor running 64-bit Fedora Core 9 Linux operating system. Only 500 samples were run due to the long run time for each sample. For demonstration purposes this was sufficient, however, many more samples would need to be run to ensure enough samples were available to fit a suitable distribution through the response. The resulting distribution for the bondline temperature is graphed in Fig. 14. According to the distribution, the probability of exceeding the bondline temperature of $315.15^{\circ} \mathrm{C}$ is $0.43 \%$, or in other words there was a $99.57 \%$ chance the bondline temperature will be below the prescribed limit. If this probability of exceeding the bondline temperature was not sufficiently low enough, the constraints of the design space must be modified and a new optimum must be determined. The designers, analysts, and mission managers are now armed with specific information as to the probability the system will perform its function under the uncertain conditions encountered during flight. If mission managers desire a 
lower probability, the most obvious constraint to be modified would be the system mass. There is now a clear picture of how the mass affects bondline temperature and its relationship to the probability of exceeding it.

The across-ply tensile stress distribution is shown in Fig. 15. Since there is no assessment of failure being made due to stress, the stress will be measured against the as-designed across-ply tensile stress. According to the distribution, the probability of exceeding the across-ply tensile stress in the as-designed compression pad is 1.88\%, or there is a $98.12 \%$ chance the stress will be below the as-designed stress. An argument similar to that of the bondline temperature can be made with the across-ply tensile stress. If the probability is not satisfactory, the design constraints would need to be modified and a new optimum determined.

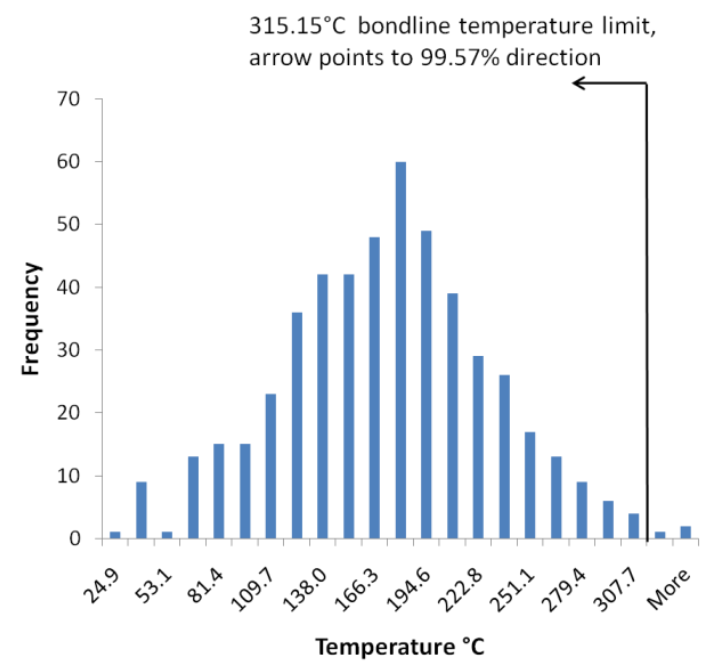

Fig. 14 Bondline temperature distribution.

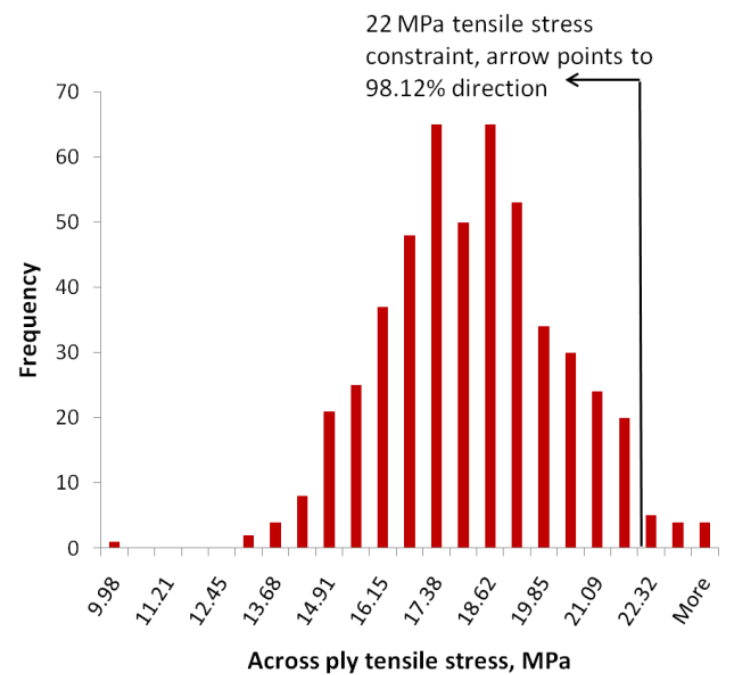

Fig. 15 Across-ply tensile stress distribution.

The design process outlined in Table 1 has been demonstrated with positive results. The link between the analysis and design has been established providing an increased knowledge about the design of the compression pad and the integrated compression pad-titanium insert system. Only a few parameters relating to the material properties were selected as the randomly varying variables in the Monte Carlo simulation. Many more parameters could have been chosen such as including uncertainty in the aerodynamic heating. Also, there are more material properties that could have been selected for screening in the property sensitivity study phase of the process. Inclusion of specific material properties, or other parameters will depend on the specific problem being solved and should be evaluated each time this design process is used. 


\section{Summary}

The FEAR program was used to demonstrate a new TPS design methodology using the CEV heatshield compression pads as a design example. The governing differential equations for ablative thermal response were presented and the finite element method was used to discretize and solve the resulting system of equations. Galerkin's weak form of the governing differential equations was presented in 3-D.

A new design approach was outlined and demonstrated by analyzing a CEV compression pad under augmented heating conditions. A link between the analysis of thermal protection systems and their design was established. The link to design comes from understanding the variation in the thermostructural response over the range of the design variables. The link between the analysis and design provides increased knowledge about the design of the compression pad and the integrated compression pad-titanium insert system.

\section{Acknowledgments}

The authors would like to thank Dr. James Reuther and Dr. Ethiraj Venkatapathy of NASA, Ames Research Center for having the confidence in the first author to lead the compression pad subsystem design effort through the programs subsystem design review while pursuing this research simultaneously. The authors would also like to thank Dr. David Schuster of NASA, Langley Research Center, Mr. Bernard Laub of NASA, Ames Research Center, Dr. Stephen Ruffin and Dr. Vitali Volovoi of Georgia Institute of Technology for their review of this work. A special thank you goes out to Dr. Ian Clark from Georgia Institute of Technology for his support of this work.

\section{References}

\footnotetext{
${ }^{1}$ Chen, Y.-K., and Milos, F. S., "Fully Implicit Ablation and Thermal Analysis Program (FIAT)," Journal of Spacecraft and Rockets, Vol. 36, No. 3, pp 475-483, May-June 1999

${ }^{2}$ Dec, J. A., Braun, R. D. and Laub, B, “Ablative Thermal Response Analysis Using the Finite Element Method", accepted for publication in the Journal of Thermophysics and Heat Transfer.

${ }^{3}$ Dec, J. A., "Three Dimensional Finite Element Ablative Thermal Response Analysis Applied to Heatshield Penetration Design," Ph.D. Dissertation, Aerospace Engineering Dept., Georgia Institute of Technology, Atlanta, GA, 2010.

${ }^{4}$ Goldstein, H. E., "Kinetics of Nylon and Phenolic Pyrolysis", Lockheed Missles and Space Company, Sunnyvale, CA. LMSC667876, October 1965.

${ }^{5}$ Goldstein, H. E., "Pyrolysis Kinetics of Nylon 6-6, Phenolic Resin, and Their Composites", Marcel Dekker, Inc., New York, 1971.

${ }^{6}$ Huebner, K. H., Dewhirst, D. L., Smith, D. E., Byrom, T. G., The Finite Element Method for Engineers, 4th ed., John Wiley \& Sons, New York, 2001.

${ }^{7}$ Reddy, J. N., Gartling, D. K., The Finite Element Method in Heat Transfer and Fluid Dynamics, 3rd ed., CRC Press, Boca Raton, pp. 175-176 2010.

${ }^{8}$ Timoshenko, S. P., Goodier, J. N., Theory of Elasticity, 3rd ed., McGraw-Hill, Inc., chapter 1-2, 1987.

9 Thompson, R.T.,"DAC-3 Computational Analysis of Beveled Compression Pad Geometries", CEV Aerosciences Project, NASA Langley Research Center, August 2009.

${ }^{10}$ Williams, A. R., and Koenig, J. R., "Rayon Replacement Program Phase IV MX4926N MT Mechanical and Thermal Characterization", SRI-ENG-07-34-9714.09, January 2007.

${ }^{11}$ Dec, J. A., and Empey, D., "Heatshield Compression Pads", Orion Thermal Protection System Subsystem Design Review, Denver, CO, July 2009.

${ }^{12}$ Anon, Tecplot 360 2009, release 2 User's Manual, Tecplot Inc., Bellevue, WA, 2009

${ }^{13}$ Dec, J.A, "Arc Jet Test Failure Analysis", Compression Pad Technical Interchange Meeting, NASA Langley Research Center, October 2008.
} 